14

\title{
Анализ электростатического взаимодействия димерных комплексов. II. Критерии и условия, предъявляемые к ингибиторам производных белка АPP
}

\author{
(C) Т.В. Кошлан, ${ }^{1}$ К.Г. Куликов ${ }^{2}$ \\ ${ }^{1}$ Санкт-Петербургский государственный университет, \\ 199034 Санкт-Петербург, Россия \\ ${ }^{2}$ Санкт-Петербургский политехнический университет Петра Великого, \\ 195251 Санкт-Петербург, Россия \\ e-mail: kulikov.kirill.g@gmail.com
}

Поступило в Редакцию 27 декабря 2019 г.

В окончательной редакции 27 декабря 2019 г.

Принято к публикации 24 января 2020 г.

\begin{abstract}
Представлены расчеты, методы подбора и даны характеристики ингибиторов образования высокомолекулярных структур амилоидными пептидами, дано объяснение их блокирующему действию. Подробно рассмотрены биофизические требования к ингибиторам, критерии и условия их отбора, которые будут блокировать образование амилоидами структур высокого порядка. В качестве ингибиторов были изучены модифицированные пептиды, которые будут образовывать с амилоидными пептидами устойчивые димеры, предотвращая тем самым вступление амилоидов во все новые биохимические реакции. Рассмотрено вступление амилоидов в биохимические реакции с себе подобными амилоидными пептидами и не будет рассмотрено их возможное реагирование со стенками сосудов. При этом в качестве критерия оценки стабильности димерного комплекса были использованы: логарифм числа обусловленности матрицы потенциальной энергии попарного электростатического взаимодействия между аминокислотными остатками и дифференциальная энтропия для случая многомерного нормального распределения.
\end{abstract}

Ключевые слова: болезнь Альцгеймера, амилоиды, ключевые аминокислотные остатки, пептиды, число обусловленности, изменение меры дифференциальной энтропии.

DOI: 10.21883/JTF.2020.08.49549.428-19

\section{Введение}

Болезнь Альцгеймера (БА) является наиболее распространенной формой деменции, которая влияет на повседневную жизнь путем потери памяти и когнитивных изменений. Ассоциация Альцгеймера сообщает, что у каждого десятого человека старше 65 лет и почти у одной трети людей старше 85 лет болезнь Альцгеймера есть. Симптомы болезни Альцгеймера обычно развиваются медленно с тенденцией постепенного ухудшения со временем, переходя от легкой забывчивости к широко распространенным нарушениям головного мозга.

До настоящего времени ни одно из проведенных III фазы клинических испытаний, нацеленных на антиБА-терапию не продемонстрировало статистически значимого эффекта лечения у пациентов с БА.

Терапевтические подходы включали в себя использование моноклональных антител, которые распознают различные эпитопы $\mathrm{A} \beta$ и проявляют различную селективность связывания [1].

Рассмотрим более подробно ранее предлагаемый для терапии БА препарат - гантенерумаб (gantenerumab).

Гантенерумаб (Gantenerumab) представляет собой полностью человеческое антитело $\mathrm{IgG1}$, предназначенное для связывания субнаномолярным сродством с конформационным эпитопом на фибриллах $A \beta$. Он охваты- вает как $N$-концевые, так и центральные аминокислоты A $\beta .19$ декабря 2014 г. компания Roche прекратила исследование на основании промежуточного анализа полезности [2].

На основании вышесказанного на сегодняшнее время существует острая необходимость в разработке новых биофизических методов патогенеза БА, в разработке новых принципов понимания ингибирования образования структур из А $\beta$-пептидов.

В качестве критериев, определяющих стабильность димерного комплекса будем использовать:

1. Меру изменения дифференциальной энтропии $(\Delta H)$, при этом $\Delta H$ есть величина безразмерная.

2. Число обусловленности матрицы, элементы которой являются потенциальные энергии электростатического взаимодействия между попарно взятыми а.а. белков. В данной физической постановке задачи оно будет характеризовать степень устойчивости конфигурации биологического комплекса. При этом для выбора более устойчивого биохимического соединения между белками выбирем матрицу потенциальной энергии электростатического взаимодействия с наименышим значением $\lg (\operatorname{cond}(W))$ (логарифм числа обусловленности матрицы потенциальной энергии попарного электростатического взаимодействия а.а.) [3-5]. 
Настоящая работа состоит из несколько частей. Разд. 1 посвящен требованиям, которые предъявляются к ингибиторам амилоидных пептидов В разд. 2 рассмотрены физические аспекты различий амилоидных пептидов с учетом двух $C$-концевых а.а. (amino acid - a.a.) ILE41 и ALA42. В заключении приведены основные выводы.

\section{1. Основные требования, предъявляемые к ингибиторам амилоидных пептидов}

В качестве модельного соединения была выбрана трехмерная структура из базы данных PDB: 2MXU [6].

Были оставлены граничащие полипептидные цепи двух амилоидных пептидов, рассчитана для них потенциальная энергия электростатического попарного взаимодействия между а.а. двух соседних цепей.

Для существенного сокращения всех вероятных вариантов возможных аминокислотных замен было выдвинуто предположение, что уменьшение пиковых значений потенциальной энергии при взаимодействии двух полипептидных аминокислотных последовательностей может приводить к снижению энтропии биологического комплекса, что, в свою очередь, может говорить о переходе биологического комплекса к более упорядоченному состоянию.

Модифицированные пептиды будем обозначать, как „Inh“, указывая номер замены а.а.. С учетом предъявляемых требований к ингибиторам амилоидных пептидов, будем изучены димеры трех типов: $\left[{ }_{w t} A \beta+{ }_{I n h} A \beta\right]$,

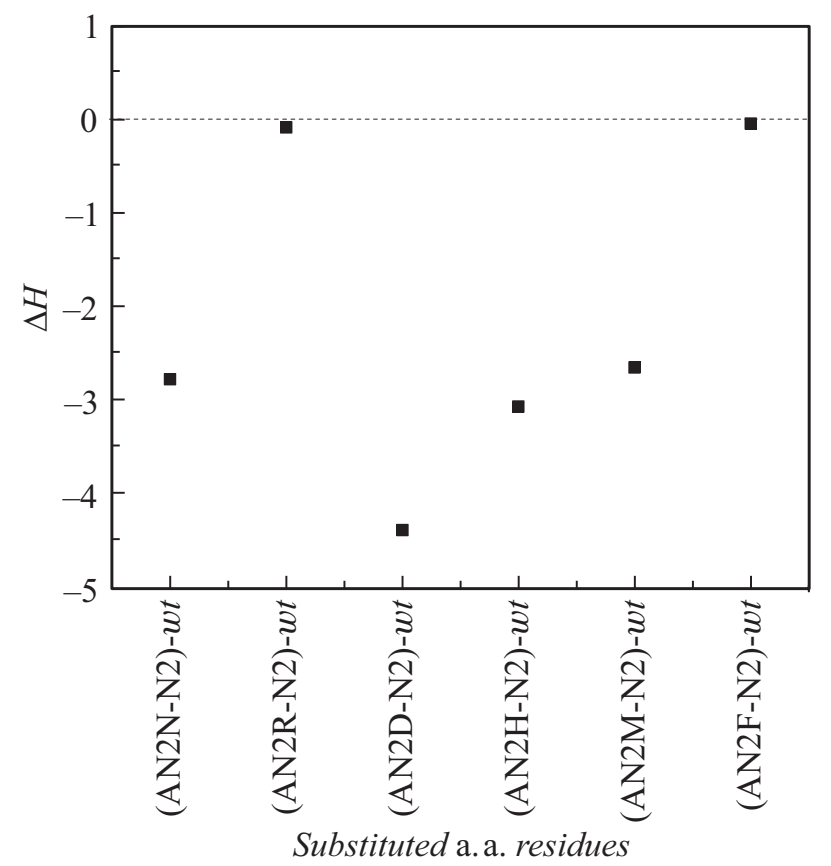

Рис. 1. Графическое представление замены а.а. N2 для амилоида $\left({ }_{w t} A \beta\right)$. Горизонтальная линия на графике определяет границу измения энтропии: область отрицательных значений от области положительных значений.

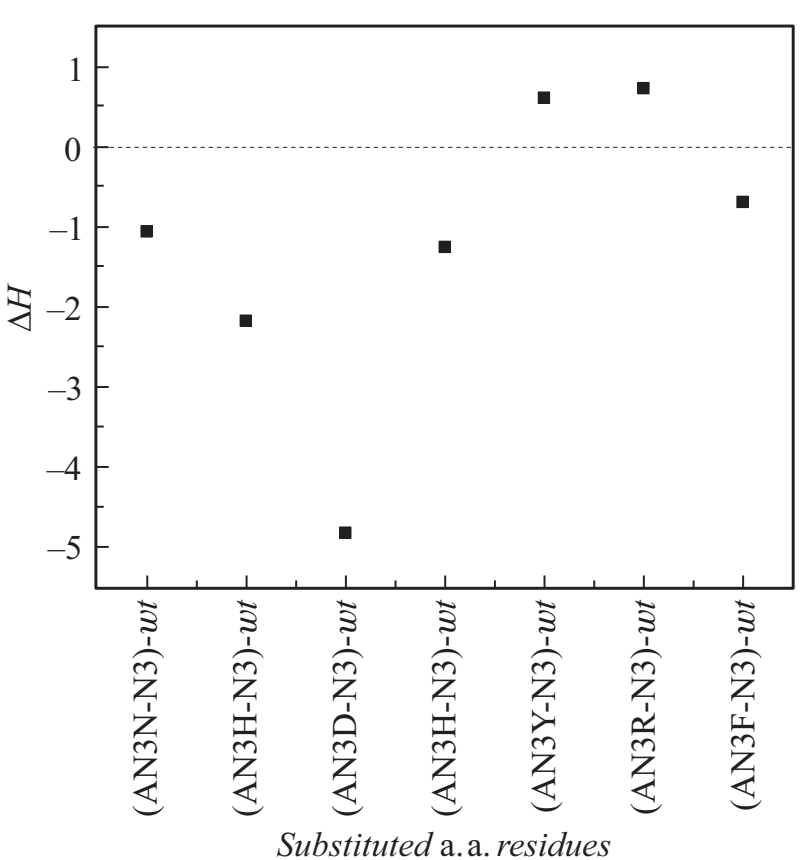

Рис. 2. Графическое представление замены аминокислотного остатка N3 для амилоида $(w t A \beta)$. Горизонтальная линия на графике определяет границу измения энтропии: область отрицательных значений от области положительных значений.

$\left[{ }_{\text {mut }} A \beta+{ }_{\text {Inh }} A \beta\right]$ и $\left[{ }_{\text {Inh }} A \beta+{ }_{\text {Inh }} A \beta\right]$. Поскольку 3-х мерная структура из PDB базы содержит аминокислотные остатки с 11-го по 42-й, то в расчетах будут учитываться исключительно петиды длиной $A \beta_{(11-42)}$, которые будут обозначены, как $A \beta$.

Ингибиторы должны характеризоваться следующими условиями связывания:

- образование устойчивого димера с амилоидным пептидом дикой формы

$\left[{ }_{w t} A \beta+{ }_{\text {Inh }} A \beta\right]$, если требуется ингибировать пептид дикой формы или образование устойчивого димера с мутантной формой амилоидного пептида $\left[{ }_{\text {mut }} A \beta+{ }_{\text {Inh }} A \beta\right]$, если требуется ингибировать амилоид мутантной формы. При этом область значений величины $\lg (\operatorname{cond}(W))$ должна находиться в диапазоне ниже значений 5.53 (в случае использования трехмерной структуры PDB:2MXU [6]);

- образование устойчивого димерного комплекса при вступлении в реакцию с таким же ингибитором $\left[{ }_{\text {Inh }} A \beta+{ }_{\text {Inh }} A \beta\right]$. При этом область значений стабильности при взаимодействии двух одинаковых ингибиторов друг с другом должна находиьтся в диапазоне значений ниже 5.53 (в случае использования трехмерной структуры PDB:2MXU [6]).

Были заменены а.а. под номерами (N2 и N3), приходящиеся на пиковые значения потенциальной энергии электростатического попарного взаимодейтсвия, в амилоиде дикого типа $\left({ }_{w t} A \beta\right)$ на а.а. (выбранные произвольным образом): $\mathrm{ASN}(\mathrm{N}), \mathrm{ARG}(\mathrm{R}), \mathrm{D}$ (ASP), H(HIS), $\mathrm{M}(\mathrm{MET}), \mathrm{F}(\mathrm{PHE})$, после этого рассчитали изменение 


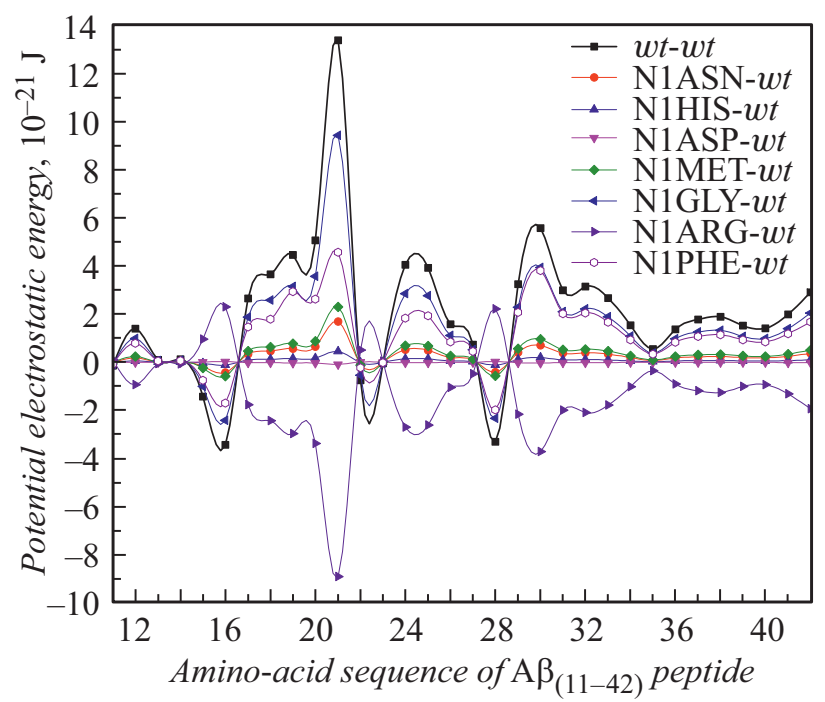

Рис. 3. Кривые потенциальной энергии электростатического взаимодействия N1-го а.а. ${ }_{t} A \beta$ со всеми остальными а.а. другого пептида с учетом замены N1-го а.а. ${ }_{t} A \beta$ последовательно на ASN, HIS, ASP, MET, GLY, ARG, PHE в одном из пептидов в димерном комплексе $\left[{ }_{w t} A \beta+{ }_{\text {Inh }} A \beta\right]$.

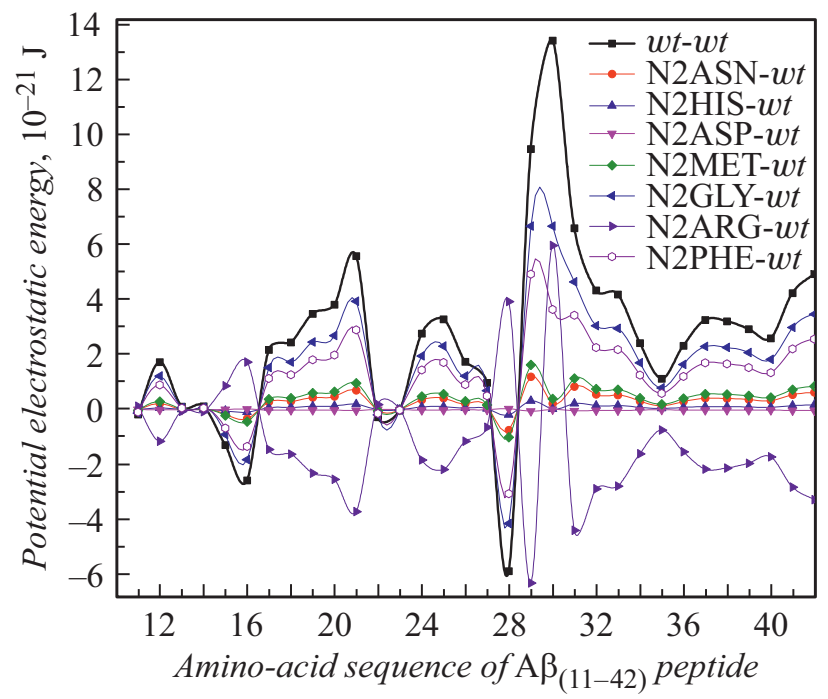

Рис. 4. Кривые потенциальной энергии электростатического взаимодействия при взаимодействии $\mathrm{N} 2$-го а.а. ${ }_{t} A \beta$ со всеми остальными а.а. другого пептида с учетом замены N2-го а.а. ${ }_{w t} A \beta$ последовательно на ASN, HIS, ASP, MET, GLY, ARG, PHE в одном из пептидов в димерном комплексе $\left[{ }_{w t} A \beta+{ }_{I n h} A \beta\right]$. Толстой линией с квадратами продемонстрировано взаимодействия а.а. N1-го и N2-го а.а. ${ }_{t} A \beta$ с пептидом дикого типа ${ }_{w t} A \beta$.

меры энтропии каждой замены для всего димерного комплекса и вычислили значения $\lg (\operatorname{cond}(W))$ для каждой замены.

Таким образом, имеется две контрольные величины: изменение меры дифференциальной энтропии $(\Delta H)$ и устойчивость биологического комплекса, который характеризуется величиной $\lg (\operatorname{cond}(W))$.
На рис. 1 представлены значения изменения меры дифференциальной энтропии между двумя димерными комплексами. В первом димерном комплексе были поочередно заменены а.а. в двух пептидах вида ${ }_{w t} A \beta$ в положении 30 на $\mathrm{ASN}(\mathrm{N}), \mathrm{ARG}(\mathrm{R}), \mathrm{D}$ (ASP), H(HIS), $\mathrm{M}(\mathrm{MET}), \mathrm{F}(\mathrm{PHE})$, второй димер был составлен из двух пептидов дикого типа $\left[{ }_{w t} A \beta\right]_{2}$.

На рис. 2 представлены значения изменения меры дифференциальной энтропии между двумя димерными комплексами ${ }_{w} A \beta$. В первом димерном комплексе были поочередно заменены аминокислотные остатки в двух пептидах в положении N3 на $\mathrm{ASN}(\mathrm{N}), \mathrm{ARG}(\mathrm{R}), \mathrm{D}(\mathrm{ASP})$, H(HIS), M(MET), F(PHE). Второй димер был составлен из двух пептидов дикого типа $\left[{ }_{w t} A \beta\right]_{2}$ (рис. 1,2$)$.

Как следует из рис. 1,2 , большинство замен N2го и N3-го а.а. для амилоида ${ }_{w t} A \beta$ привели к отрицательным значениям изменения меры энтропии $(\Delta H)$ кроме двух случаев: $\left[\left({ }_{\operatorname{Inh}} A \beta_{(N 3 Y)}-{ }_{w t} A \beta\right)-\left({ }_{w t} A \beta\right)_{2}\right]$ и $\left[\left({ }_{\operatorname{Inh}} A \beta_{(N 3 R)}-{ }_{w t} A \beta\right)-\left({ }_{w t} A \beta\right)_{2}\right]$. При этом мера изменения дифференциальной энтропии димерных комплексов увеличилась при переходе к взаимодействию диких форм амилоидных пептидов от мутантных форм.

Анализ графиков (рис. 1 и 2) позволяет сделать вывод, что замена N2-го и N3-го а.а. ${ }_{t} A \beta$ на различные а.а. по разному влияет на изменение диффреренциальной энтропии.

Посмотрим, какое влияние замены N1-го, N2-го и $\mathrm{N} 3$-го а.а. ${ }_{t} A \beta$ оказывает на изменение потенциальной энергии электростатического взаимодействия со всеми остальными а.а. полипептидной цепи второго амилоидного пептида ${ }_{w t} A \beta$ (рис. 3-5).

Анализ графиков (рис. 3-5) позволяет сделать вывод, что замена N1-го, N2-го и N3-го а.а. ${ }_{w} A \beta$ на ASN,HIS,

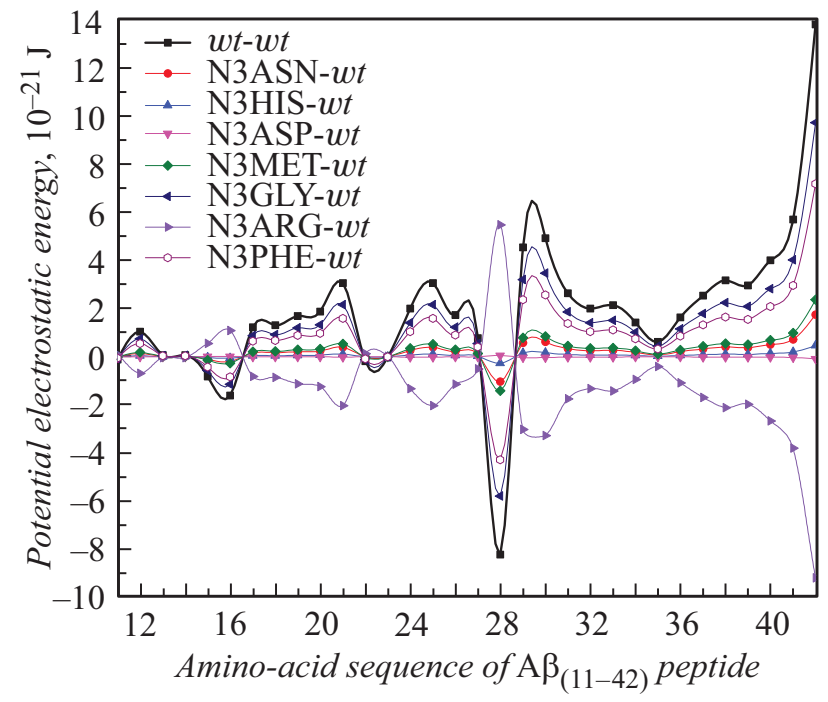

Рис. 5. Кривые потенциальной энергии электростатического взаимодействия при взаимодействии $\mathrm{N} 3$-го а.а. ${ }_{t} A \beta$ со всеми остальными а.а. другого пептида с учетом замены N3-го а.а. ${ }_{w t} A \beta$ последовательно на ASN, HIS, ASP, MET, GLY, ARG, PHE в одном из пептидов в димерном комплексе $\left[{ }_{w t} A \beta+{ }_{I n h} A \beta\right]$. 


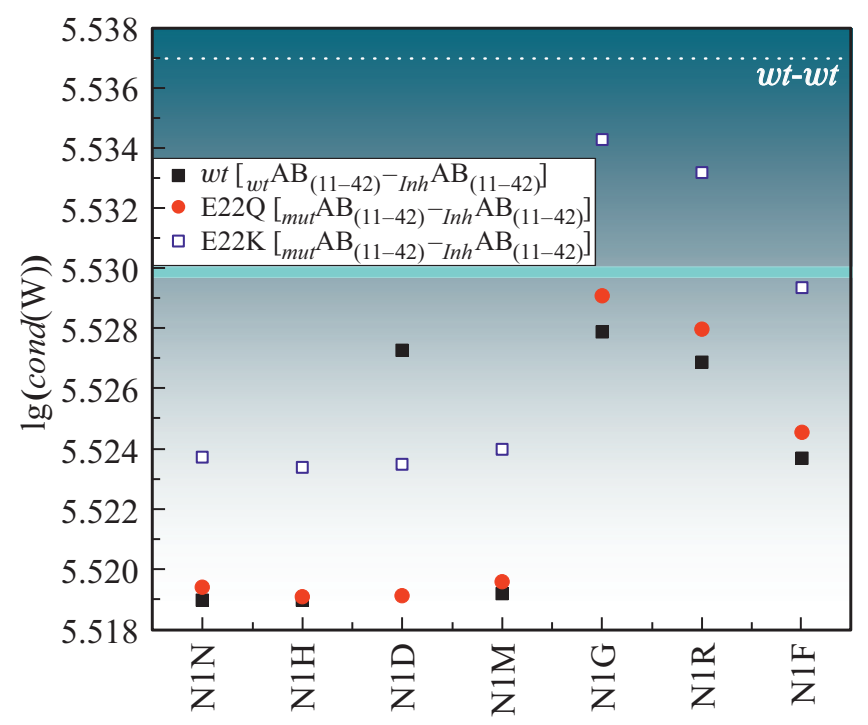

Substituted a. a. residues

Рис. 6. Графическое представление численных значений стабильности взаимодействия модифицированного пептида ${ }_{\text {Inh }} A \beta$ (замена N1-го а.а. $\left.{ }_{w t} A \beta\right)$ c ${ }_{w t} A \beta$ (черный квадратик), с мутантной формой тит $A \beta_{(E 22 Q)}$ (кружочек), с мутантной формой ${ }_{m u t} A \beta_{(\text {Е22K) }}$ (пустой квадратик). Обозначение „АВ“ означает амилоидный пептид $A \beta$.

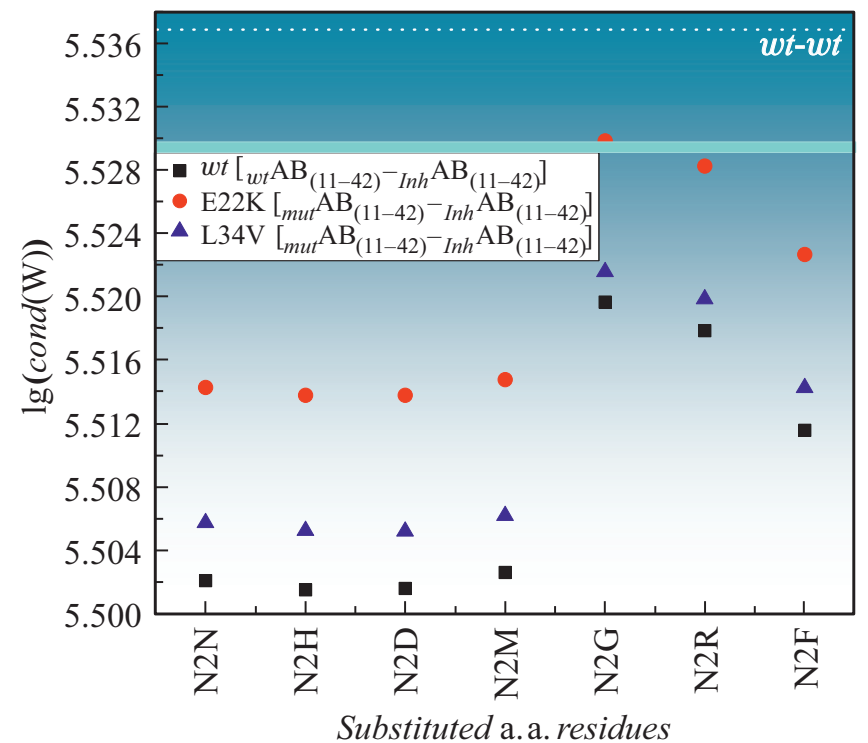

Рис. 7. Графическое представление численных значений стабильности взаимодействия модифицированного пептида ${ }_{\operatorname{Inh}} A \beta$ (замена N2-го а.а. ${ }_{t} A \beta$ ) с амилоидным пептидом ${ }_{t} A \beta$ (черный квадратик), с мутантной формой тит $A \beta_{(E 22 K)}$ (кружочек), с мутантной формой тиt $A \beta_{(L 34 V)}$ (треугольник). Белой пунктирной линией обозначена численная величина взаимодействия $\left[{ }_{w} A \beta\right]_{2}$ Обозначение „АВ“ означает амилоидный пептид $A \beta$.

ASP,MET, GLY, ARG, PHE приводит к уменьшению значений энергии.

Перейдем к расчету и анализу значений $\lg (\operatorname{cond}(W))$ димерных комплексов с выполненными заменами а.а. в пиковых значениях потенциальной энергии электростатического взаимодействия для случаев $\left[{ }_{w t} A \beta+{ }_{I n h} A \beta\right]$ и $\left[{ }_{\text {mut }} A \beta+{ }_{\text {Inh }} A \beta\right]$.

Начнем с анализа замены N1-го, N2-го а.а. ${ }_{w} A \beta$. Ниже представлены графики (рис. 6-8), которые позволяют определить какие замены а.а. приведут к увеличению

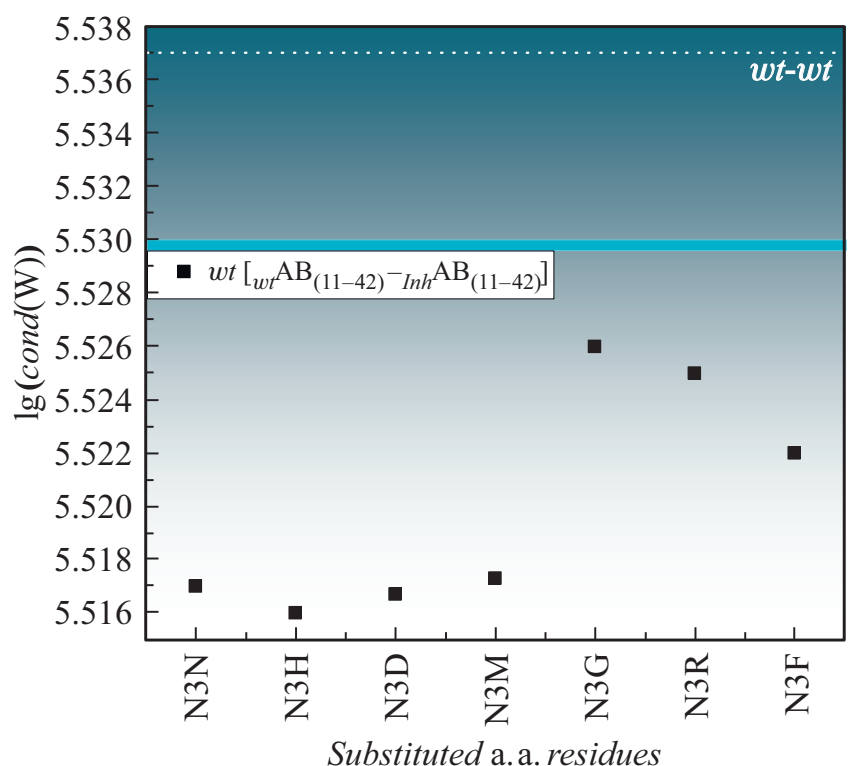

Рис. 8. Графическое представление численных значений стабильности взаимодействия модифицированного пептида $\operatorname{Inh} A \beta$ (замена N3-го а.а. ${ }_{w} A \beta$ ) с амилоидным пептидом ${ }_{w t} A \beta$ (черный квадратик).Белой пунктирной линией обозначена численная величина взаимодействия $\left[{ }_{w t} A \beta\right]_{2}$. Обозначение „АВ“ означает амилоидный пептид $A \beta$.

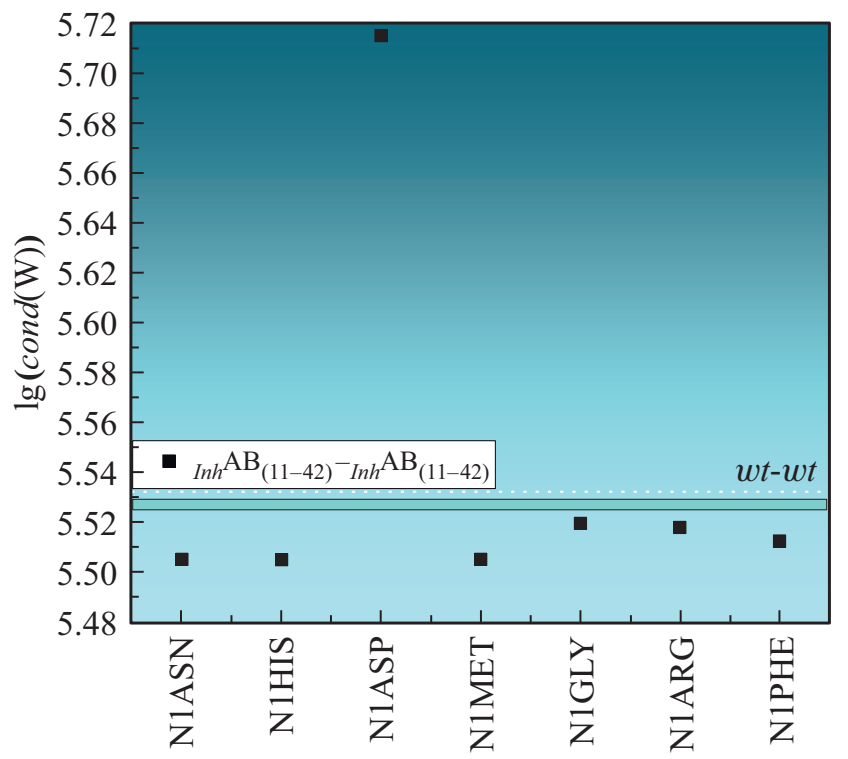

Substituted a. a. residues

Pис. 9. График, харктеризующий устойчивость модифицированных димерных комплексов $\left[{ }_{i n h} A \beta-{ }_{i n h} A \beta\right]$ при замене N2-го a.а. последовательно на ASN, HIS, ASP, MET, GLY, ARG, PHE. Обозначение „АВ“ означает амилоидный пептид $A \beta$. 


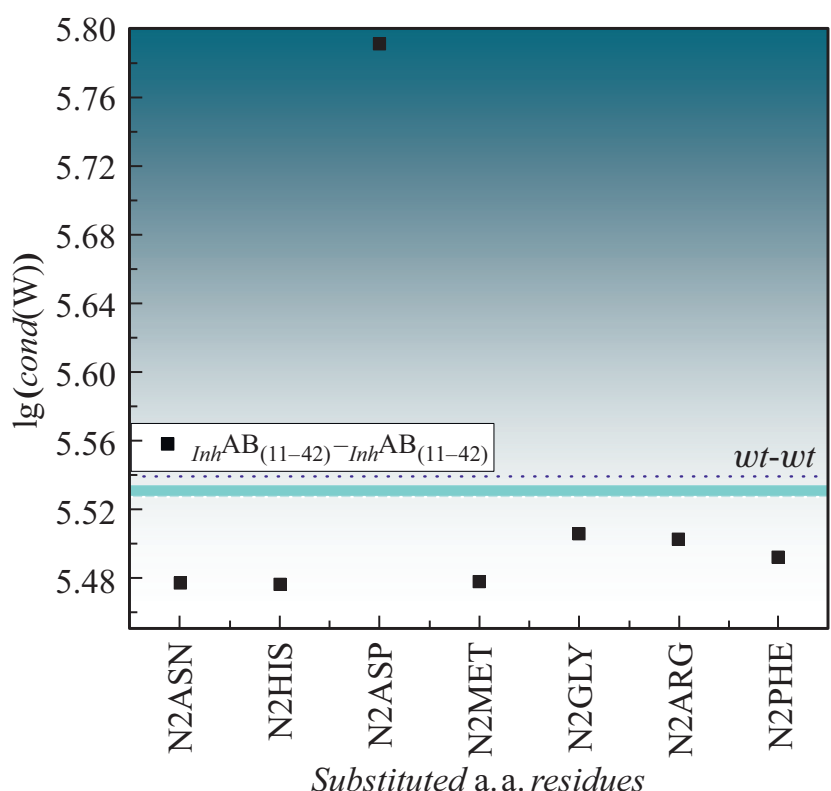

Рис. 10. График, харктеризующий устойчивость модифицированных димерных комплексов $\left[{ }_{i n h} A \beta-{ }_{i n h} A \beta\right]$ при замене N1-го a.a. последовательно на ASN, HIS, ASP, MET, GLY, ARG, PHE. Обозначение „АВ“ означает амилоидный пептид $A \beta$.

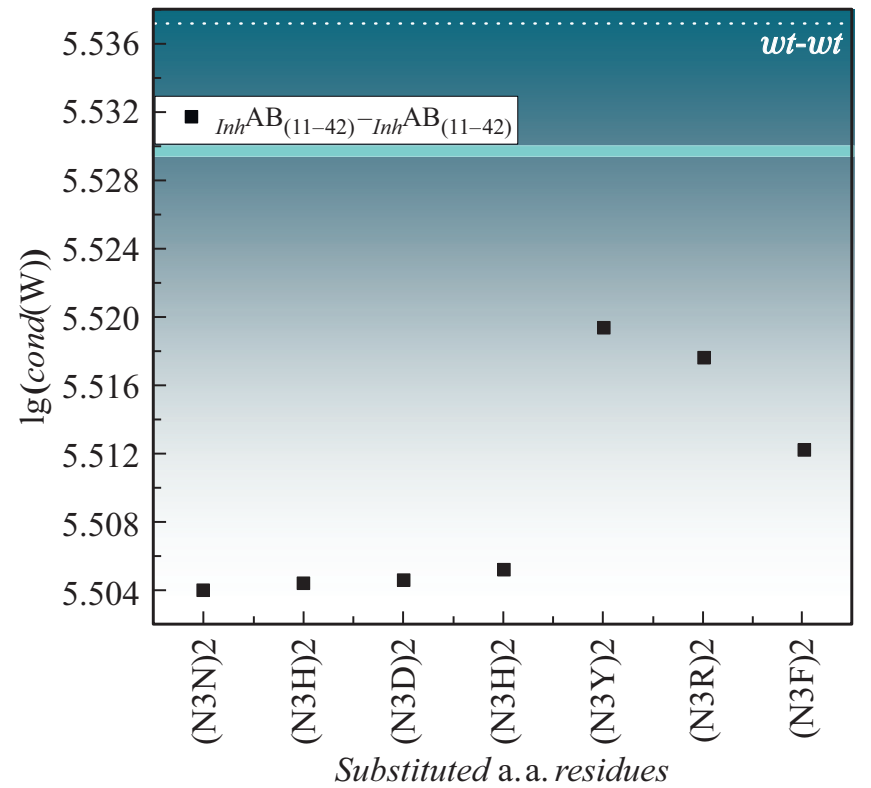

Pис. 11. График, харктеризующий устойчивость модифицированных димерных комплексов $\left[{ }_{i n h} A \beta-{ }_{i n h} A \beta\right]$ при замене N3-го a.а. последовательно на ASN, HIS, ASP, MET, GLY, ARG, PHE. Обозначение „АВ“ означает амилоидный пептид $A \beta$.

стабильности димерных комплексов при взаимодействии с ${ }_{w t} A \beta$ и с ${ }_{m u t} A \beta$.

При этом линией на уровне 5.53 указано условное разделение численных результатов на две области: область численных значений, отвечающих менее стабильным димерам (над чертой), область численных значений, ха-
Таблица 1. Перечень замен аминокислотных остатков

\begin{tabular}{|c|c|c|}
\hline $\begin{array}{c}\text { Наименование } \\
\text { амилоида }\end{array}$ & $\begin{array}{c}\text { Номер } \\
\text { а.а. }\end{array}$ & a.a. \\
\hline wt $A \beta$ & $\begin{array}{l}\text { N1 } \\
\text { N2 } \\
\text { N3 }\end{array}$ & 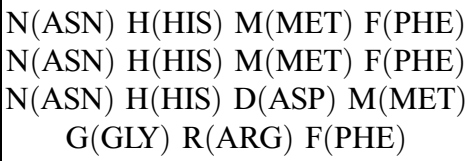 \\
\hline $\begin{array}{c}{ }_{m u t} A \beta_{(E 22 Q)} \\
E 22 Q(G L U 22 G L N)\end{array}$ & N1 & $\mathrm{N}(\mathrm{ASN}) \mathrm{H}(\mathrm{HIS}) \mathrm{M}(\mathrm{MET}) \mathrm{F}(\mathrm{PHE})$ \\
\hline $\begin{array}{c}{ }_{m u t} A \beta_{(E 22 Q)} \\
\mathrm{E} 22 \mathrm{Q}(\mathrm{GLU} 22 \mathrm{GLN})\end{array}$ & $\begin{array}{l}\text { N1 } \\
\text { N2 }\end{array}$ & $\begin{array}{c}\mathrm{N}(\mathrm{ASN}) \mathrm{H}(\mathrm{HIS}) \mathrm{M}(\mathrm{MET}) \\
\mathrm{N}(\mathrm{ASN}) \mathrm{H}(\mathrm{HIS}) \mathrm{M}(\mathrm{MET}) \mathrm{F}(\mathrm{PHE})\end{array}$ \\
\hline $\begin{array}{c}{ }_{m u t} A \beta_{(L 34 V)} \\
\text { L34V(LEU34VAL })\end{array}$ & $\mathrm{N} 2$ & $\mathrm{~N}(\mathrm{ASN}) \mathrm{H}(\mathrm{HIS}) \mathrm{M}(\mathrm{MET}) \mathrm{F}(\mathrm{PHE})$ \\
\hline
\end{tabular}

рактеризующих более стабильные димерные комплексы (под чертой).

Перейдем к рассмотрению полученных результатов при замене N1-го и N2-го а.а. в амилоидном пептиде Inh $A \beta$ при взаимодействии с ${ }_{w t} A \beta$ и с пептидами тит $A \beta$ (рис. 9-11).

Наилучшие результаты были получены при замене N1-го и N2-го а.а. в амилоидном пептиде на а.a. ASN, ASP, HIS, MET. Данные замены в наибольшей степени способствовали увеличению стабильности димерного комплекса при участии модифицированного пептида ${ }_{i n h} A \beta$ и амилоидного пептида как дикой формы ${ }_{w t} A \beta$, так и мутантных форм тит $A \beta$. Значения показателя, характеризующего устойчивое состояние приведенных димеров, располагаются в нижней области значений величины $\lg (\operatorname{cond}(W))$.

Bсе выполненные замены N3-го а.а. на ASN, HIS, ASP, MET, GLY, ARG, PHE в модифицированных пептидах при взаимодействии с ${ }_{w t} A \beta$ приводят к увеличению устойчивости димерного комплекса. При этом значения показателя, характеризующего устойчивое состояние приведенных димеров, располагаются в нижней области значений величины $\lg (\operatorname{cond}(W))$ (рис. 9-11).

При замене N1-го и N2-го а.а. на аспарагиновую кислоту (ASP) при взаимодействии двух одинаково модифицированных пептидов $\left[{ }_{i n h} A \beta-{ }_{i n h} A \beta\right]$ происходит резкое увелечение величины $\lg (\operatorname{cond}(W))$, характеризующее устойчивость димерного комплекса (рис. 9-10).

Во всех заменах N3-го а.а. на ASN, HIS, ASP, MET, GLY, ARG, PHE наблюдается увеличение стабильности димерных комплексов, образованных двумя пептидными ингибиторами (рис.11).

Совместный анализ попарных графиков (рис. 6-9, 7-10 и 8-11) позволяет определить модифицированные пептиды, которые в наибольшей степени склонны образовывать стабильные димеры как с амилоидными пептидами, так и при взаимодействии с собой. 
Таблица 2. Значения потенциальной энергии электростатического взаимодействия $C$-концевых участков амилоидных пептидов

\begin{tabular}{l|r|r|r|r|r|r|r}
\hline & VAL36 & GLY37 & GLY38 & VAL39 & VAL40 & ILE41 & ALA42 \\
\cline { 2 - 7 } ILE31 & $1.5 \cdot 10^{-21}$ & $1.7 \cdot 10^{-21}$ & $1.7 \cdot 10^{-21}$ & $1.3 \cdot 10^{-21}$ & $1.1 \cdot 10^{-21}$ & $1.4 \cdot 10^{-21}$ & $1.9 \cdot 10^{-21}$ \\
GLY33 & $2.2 \cdot 10^{-21}$ & $2.4 \cdot 10^{-21}$ & $2.3 \cdot 10^{-21}$ & $1.6 \cdot 10^{-21}$ & $1.3 \cdot 10^{-21}$ & $1.6 \cdot 10^{-21}$ & $2.1 \cdot 10^{-21}$ \\
LEU34 & $2.3 \cdot 10^{-21}$ & $2.1 \cdot 10^{-21}$ & $1.9 \cdot 10^{-21}$ & $1.2 \cdot 10^{-21}$ & $9.3 \cdot 10^{-22}$ & $1.1 \cdot 10^{-21}$ & $1.4 \cdot 10^{-21}$ \\
MET35 & $9.8 \cdot 10^{-22}$ & $1 \cdot 10^{-21}$ & $1 \cdot 10^{-21}$ & $6.5 \cdot 10^{-22}$ & $4.5 \cdot 10^{-22}$ & $5.1 \cdot 10^{-22}$ & $6.3 \cdot 10^{-22}$ \\
VAL36 & $3.6 \cdot 10^{-21}$ & $3.4 \cdot 10^{-21}$ & $2.8 \cdot 10^{-21}$ & $1.6 \cdot 10^{-21}$ & $1.2 \cdot 10^{-21}$ & $1.3 \cdot 10^{-21}$ & $1.6 \cdot 10^{-21}$ \\
GLY37 & $4.2 \cdot 10^{-21}$ & $6.7 \cdot 10^{-21}$ & $6.7 \cdot 10^{-21}$ & $3 \cdot 10^{-21}$ & $2.1 \cdot 10^{-21}$ & $2.1 \cdot 10^{-21}$ & $2.6 \cdot 10^{-21}$ \\
GLY38 & $2.6 \cdot 10^{-21}$ & $4.8 \cdot 10^{-21}$ & $6.7 \cdot 10^{-21}$ & $3.5 \cdot 10^{-21}$ & $2.7 \cdot 10^{-21}$ & $2.4 \cdot 10^{-21}$ & $3.1 \cdot 10^{-21}$ \\
VAL39 & $1.6 \cdot 10^{-21}$ & $2.8 \cdot 10^{-21}$ & $4.4 \cdot 10^{-21}$ & $3.6 \cdot 10^{-21}$ & $2.7 \cdot 10^{-21}$ & $2.4 \cdot 10^{-21}$ & $2.9 \cdot 10^{-21}$ \\
VAL40 & $1.2 \cdot 10^{-21}$ & $2 \cdot 10^{-21}$ & $2.8 \cdot 10^{-21}$ & $2.6 \cdot 10^{-21}$ & $3.4 \cdot 10^{-21}$ & $2.7 \cdot 10^{-21}$ & $3.8 \cdot 10^{-21}$ \\
ILE41 & $1.3 \cdot 10^{-21}$ & $2.1 \cdot 10^{-21}$ & $2.8 \cdot 10^{-21}$ & $2.9 \cdot 10^{-21}$ & $3.7 \cdot 10^{-21}$ & $5.4 \cdot 10^{-21}$ & $6.7 \cdot 10^{-21}$ \\
ALA42 & $1.6 \cdot 10^{-21}$ & $2.5 \cdot 10^{-21}$ & $3.2 \cdot 10^{-21}$ & $3 \cdot 10^{-21}$ & $4 \cdot 10^{-21}$ & $5.7 \cdot 10^{-21}$ & $1.4 \cdot 10^{-20}$
\end{tabular}



Рис. 12. Трехмерное представление потенциальной энергии электростатического попарного взаимодействия между $C$-концевыми a.a. ILE41(I41), ALA42(A42), LYS28(K28) амилоидных пептидов.

В табл. 1 приведены замены а.а., полученные в ходе модельного эксперимента, которые подходят в качестве блокаторов высокомолекулярных структур амилоидов.

При этом стоит отметить, что был проведен анализ не целого амилоидного пептида, а участка, представленного в 3-х мерной структуре PDB с 11-го по 42-й а.а.. Предполагается, что для создания блокирующих ингибиторов следует уделить внимание также и взаимодействиям с 1-го по 10-й а.а. в амилоидном пептиде.

Также следует отметить, что по мере формирования олигомеров, протофибрилл и фибрилл, амилоидные пептиды постепенно изменяют свою структуру образования высокомолекулярных комплексов. При этом одной из причин постоянно меняющейся трехмерной структуры присоединения все новых амилоидных пептидов объясняется попыткой амилоидного конгломерата достичь устойчивого биохимического состояния.

\section{2. Физические аспекты различий амилоидных пептидов с учетом двух C-концевых а.а. ILE41 и ALA42}

Накопившиеся в литературе данные указывают на различия в физических аспектах взаимодействий амилоидных пептидов $A \beta_{(1-40)}$ и $A \beta_{(1-42)}$, однако на данный момент отсутствуют четкие причины этих отличий.

Хотя эти два вида $A \beta_{(1-40)}$ и $A \beta_{(1-42)}$ отличаются только двумя а.а., последние исследования показывают, что они значительно различаются по метаболизму, физиологическим функциям, токсичности и механизму агрегации [6-10].

Хотя в целом принято считать, что немутантный $A \beta_{42}$ более токсичен, чем немутантный $A \beta_{40}$, объяснение его предположительно повышенной скорости агрегации $A \beta_{42} / A \beta_{40}$ [11] остается неясным [7,12-17]. 
В данной работе представлена новая трактовка, которая поможет прояснить отличия в физических аспектах между этими двумя вариантами амилоидных пептидов $A \beta_{(1-40)}$ и $A \beta_{(1-42)}$.

Приведем участок таблицы потенциальной энергии электростатического взаимодействия с указанием пиковых значений потенциальной энергии (табл. 2).

Цветом в таблице выделены максимальные значения потенциальной энергии электростатического попарного взаимодействия. На область $C$-конца приходится повышенная „Плотность“ высоких значений величины потенциальной энергии.

Участки взаимодействия а.a. ILE41 и ALA42 характеризуются большими значения потенциальной энергии при взаимодействии с аналогичными а.а. соседнего пептида с пиковым значением в положительной области значений, равным $1.4 \cdot 10^{-20} \mathrm{~J}$. Соответствующее трехмерное представление потенциальной энергии электростатического взаимодействия приведено на рис. 12 с указанием пиковых значений.

У амилоидного пептида $\mathrm{A} \beta_{(11-40)}$ соответствующие пиковые значения отсутствуют.

Вычислим меру измения дифференциальной энтропии между двумя димерными комплексами, образованными пептидами ${ }_{w t} A \beta_{(11-40)}$ и ${ }_{w t} A \beta_{(11-42)}$. Воспользуемся для этого данными, приведенных в табл. 2 в области $C$-конца двух пептидов.

В этом случае мера изменения энтропии $(\Delta H)$ будет равна 94.1 при переходе из системы, содержащей ILE40(I40) и ALA42 (A42), к системе, оканчивающейся на а.a. VAL40(V40).

Таким образом, может быть сделан вывод о том, что участие а.a. ILE41(I41) и ALA42(A42) амилоидных пептидов приводит к более неустойчивому димерному комплексу $\left[{ }_{w t} A \beta_{(11-42)}\right]_{2}$ по сравнению с $\left[{ }_{w t} A \beta_{(11-40)}\right]_{2}$.

\section{Выводы}

В работе был рассмотрен новый метод, который позволит по-новому взглянуть на проблему агрегации амилоидных пептидов и разрешит получать качественную оценку способности амилоидных пептидов образовывать структуры более высокого порядка.

Перечислены физические требования к ингибиторам амилоидных пептидов, дабы предотвратить образование высокомолекулярных структур.

На основе разработанного метода предложен метод блокировки вступления амилоидных пептидов в реакции с себе подобными пептидами путем достижения устойчивого состояния амилоидных пептидов на уровне димерных комплексов.

Так, ингибиторы должны образовывать димерные комплексы с амилоидными пептидами, при этом контроль стабильности димерного комплекса предлагается осуществлять путем анализа меры изменения энтропии и величины $\lg (\operatorname{cond}(W))$.
При этом димерный комплекс должен характеризовать величиной $\lg (\operatorname{cond}(W))$ со значением ниже 5.53 (если в расчетной модели используется 3-х мерная структура из PDB-базы 2MXU [6]).

Согласно нашим предположениям, наиболее значимое изменение в аффинности двух димерных комплексах будут привносить замены а.а., на которые приходятся наибольшие значения потенциальной энергии („ключевых ${ }^{6}$ а.а.).

Дальнейшие единичные замены „ключевых“ $а$ а.а. позволили снизить величину $\lg (\operatorname{cond}(W))$ взаимодействующих пептидов ниже отметки 5.53, которая является условной разделяющей границей между более устойчивыми и менее устойчивыми димерами.

Поскольку модифицированные пептиды могут взаимодействовать и между собой, то следует проверять также их устойчивость в образовании гомодимерных комплексов.

Величина $\lg (\operatorname{cond}(W))$ для гомодимерных модифицированных пептидов также не должна превышать значения 5.53 при условии, что в качестве расчетной 3-х мерной модели берется структура из PDB-базы: 2MXU [6].

Таким образом, разработанный в работе подход на основе введенных критериев: мера изменения дифференциальной энтропии и число обусловленности матрицы, элементы которой являются потенциальные энергии электростатического взаимодействия между попарно взятыми а.а. белков, дает возможным блокировать вступление амилоидных пептидов в биохимические реакции.

\section{Конфликт интересов}

Авторы заявляют, что у них нет конфликта интересов.

\section{Список литературы}

[1] Budd Haeberlein S., O'Gorman J., Chiao P., Bussiere T., von Rosenstiel P., Tian Y., Zhu Y., von Hehn C., Gheuens S., Skordos L., Chen T., Sandrock A. // J. Prev. Alzheimers Dis. 2017. Vol. 4. N 4. P. 255-263.

[2] URL:https://www.alzforum.org/therapeutics/gantenerumab (дата обращения 6.12.2019)

[3] Koshlan T.V., Kulikov K.G. Mathematical ModelInh of Protein Complexes. SprInher: Nature, 2018. 367 p.

[4] Кошлан Т.В., Куликов К.Г. // ЖТФ. 2018. Т. 88. Вып. 8. C. 1137-1149.

[5] Кошлан Т.В., Куликов К.Г. // ЖТФ. 2018. Т. 88. Вып. 8. C. 1150-1159.

[6] Protein data bank - URL:https://www.rcsb.org/(дата обращения 6.12.2019).

[7] Qiu T., Liu Q., Chen Y.X., Zhao Y.F., Li Y.M. A $\beta 42$ and $A \beta 40$ : similarities and differences // SpecialIssue: 13th Chinese International Peptide Symposium. 2015. Vol. 21. N 7. P. 522-529.

[8] Glenner G.G., Wong C.W. // Biochem Biophys. Res. Commun. 1984. Vol. 120. N 3. P. 885-890.

[9] Hardy J., Allsop D. // Trends Pharmacol. Sci. 1991. Vol. 12. N 10. P. 383-388. 
[10] Selkoe D., Hardy J. // EMBO Mol. Med. 2016. Vol. 8. N 6. P. 595-608.

[11] Cohen S.I., Linse S., Luheshi L.M., Hellstrand E., White D.A., Rajah L., Otzen D.E., Vendruscolo M., Dobson C.M., Knowles T.P. // Proc. Natl. Acad. Sci. USA. 2013. Vol. 110. N.24. P. $9758-9763$.

[12] Sandberg A., Luheshi L.M., Sollvander S., Pereira de Barros T. // Proc. Natl. Acad. Sci. USA. 2010. Vol. 107. N 35. P. 15595-155600.

[13] Benilova I., Karran E., De Strooper B. // Nature Neurosci. 2012. Vol. 15. N 3. P. 349-357.

[14] Wei H., Schulten K. // J. Am. Chem. Soc. 2014. Vol. 136. N 35. P. 2450-12460.

[15] Yang X., Meisl G., Frohm B., Thulin E., Knowles T.P.J., Linse S. // Proc. Natl. Acad. Sci. USA. 2018. Vol. 115. N 26. P. E5849-E5858.

[16] Hsu F., Giovanna Park G., Gu Z. // ACS. Omega. 2018. Vol. 3. N 7. P. 84018407.

[17] Phillips J.C. // ACS Chem. Neurosci. 2019. Vol. 10. N 6. P. 2843-2847. 